\title{
The Effect of Presence of Helicobacter pylori on the Severity and Clinical Course of Rosacea
}

\author{
Đuka Ninković Baroš, ${ }^{1,2}$ Vesna Gajanin, ${ }^{2}$ Nevena Kutlija, ${ }^{3}$ Igor Sladojević, ${ }^{2}$ \\ Svetozar Krivokuća ${ }^{1}$
}

\begin{abstract}
Introduction: Rosacea is manifested by erythema and telangiectasias limited to the regions of cheeks, nose, chin and forehead. Data from the literature indicate a possible causal relationship between Helicobacter pylori infection in the gastrointestinal tract and rosacea, which is confirmed by the improvement of symptoms and clinical picture of rosacea after administered Helicobacter pylori eradication therapy. The aim of the paper was to determine the frequency of Helicobacter pylori infection in patients with rosacea and to examine the effect of the therapy for eradication of infection of this microorganism on the clinical course of rosacea.

Methods: Sixty patients with a diagnosis of rosacea who were treated in 2018 at the Clinic for Skin and Venereal Diseases of the University Clinical Centre of Republic of Srpska were analysed. Patients were examined during their visit to a dermatologist (first week) and after applied therapy (sixth week). Subjects were divided into two groups - group I consisted of subjects in which, in addition to rosacea, the presence of Helicobacter pylori was registered and for whom the topical therapy and triple therapy for eradication of Helicobacter pylori were administered, and group II, which included subjects in which, in addition to rosacea, the presence of Helicobacter pylori was not registered and for whom only topical therapy was administered. Statistical processing was performed in the IBM SPSS Statistics 21 software package.

Results: Helicobacter pylori infection was registered in $45 \%$ of patients. Statistical significance was determined between the presence of Helicobacter pylori infection and the presence of pustules in patients with rosacea ( $p=0.027)$, with an occurrence of pustules significantly more frequent in patients belonging to group I (55.6\%) than in patients belonging to group II (24.2\%).

Conclusion: Therapy for eradicating Helicobacter pylori infection improves the clinical course of rosacea, especially in the stage of papules and pustules.
\end{abstract}

Key words: Rosacea; Clinical course; Helicobacter pylori, effect of medications;
(1) University Clinical Centre of the Republic of Srpska, Banja Luka, the Republic of Srpska, Bosnia and Herzegovina.

(2) Faculty of Medicine, University of Banja Luka, Banja Luka, the Republic of Srpska, Bosnia and Herzegovina.

(3) Alfalab laboratory, Banja Luka, the Republic of Srpska, Bosnia and Herzegovina.

Correspondence:

ĐUKA NINKOVIĆ BAROŠ

E: djukaninkovic@yahoo.com

\section{ARTICLE INFO}

Received: 2 December 2020 Revision received: 18 August 2021 Accepted: 18 August 2021

\section{Introduction}

Rosacea (lat. Acne rosace) is a chronic skin disease the basic characteristics of which include erythema of the central face regions with the appearance of papules and pustules as well as hyperplasia of the connective tissue of the skin and sebaceous glands. ${ }^{1}$ It attacks both sexes equally, although in women, aged 40-50 years, the disease occurs more often with milder symptoms. ${ }^{2}$ Several factors play a role in the development of the pathological process in this disease: skin vasculature, digestive 
tract disorders, endocrine factors, immune and psychosomatic disorders. The available literature points at a potential link between the Helicobacter pylori (HP) infection and rosacea. ${ }^{3}$ The first report of a possible link between HP and rosacea dates back to 1994 . Thirty-one patient with rosacea participated in that study, and $84 \%$ of them were found to have HP infection. After metronidazole therapy, the symptoms of rosacea either receded or disappeared completely. ${ }^{4}$ In the sample of a study by Agnolleti et al, HP infection was present in $88 \%$ of patients with rosacea, ${ }^{5}$ while a study by Bhattarai et al, gastric HP infection in patients with rosacea was present in $65.4 \%$ of patients. ${ }^{6}$ Since HP infection is associated with the symptoms and skin changes in patients with rosacea, several studies proved the improvement in the symptoms and clinical picture of rosacea following HP eradication therapy. ${ }^{7-9}$

The clinical picture of rosacea is characterised by persistent erythema and telangiectasias limited to the regions of cheeks, nose, chin and forehead, involving, rarely, the entire surface of the face with oedema or erythematous papules and/ or pustules. ${ }^{10,11}$ The diagnosis of rosacea is established on the basis of a well-taken anamnesis and clinical picture. Digital dermatoscopy is also used in the diagnostics with an obligatory test for the presence of Demodex folliculorum mites and a test for the presence of HP infection. ${ }^{12}$ Non-invasive methods in the diagnostics of HP include the detection of its specific activity (urease enzyme - urease breath test), the presence of specific antibodies in the saliva and/or serum of an infected person or the detection of HP antigen in the patient's stool. Invasive methods reveal the presence of HP (microbiological examination, histological examination) or its activity (rapid urease test) in biopsy samples of gastric mucosa obtained by endoscopic examination. ${ }^{13}$ The differential diagnosis largely depends on the form of rosacea, with the exclusion of seborrheic dermatitis, lupus erythematosus and other photodermatoses, carcinoid syndrome and mitral valve insufficiency, acne, bromoderma and iododerma, perioral dermatitis and pustular folliculitis, lupus vulgaris and cutaneous sarcoidosis. ${ }^{14,15}$ Classic therapy of rosacea is based on the use of antibacterial drugs from the group of macrolides and metronidazole and topical therapeutic agents, and in more severe forms, also, of synthetic retinoids or combined therapeutic modalities, as in the case of eradication of Demodex folliculorum mites with anti-inflammatory therapy and phys- iotherapy, low-power and high-power laser methods, and photodynamic therapy. ${ }^{16-19}$

The aim of this study was to determine the frequency of HP infection in patients with rosacea as well as to examine the effect of the therapy for HP infection eradication on the clinical course of rosacea.

\section{Methods}

In a prospective study, 60 patients diagnosed with rosacea who were treated in 2018 at the Clinic for Skin and Venereal Diseases of the University Clinical Centre of Republic of Srpska were analysed. Only patients diagnosed with rosacea over the age of 18 were included in the study, while patients under the age of 18 and patients who did not adhere to the treatment protocol and did not come for weekly follow-up examinations were excluded from the study.

Patients were referred to a dermatologist for purposes of assessment of the subjects' skin phototype according to Fitzpatrick ${ }^{20}$ and the presence of general symptoms in the region of the affected skin (burning, itching and pain). The dermatological status of the skin affected by the changes was determined, ie, the gradation of the intensity of the disease was performed, from the mildest to the most severe stages: erythema, papules, pustules and phyma, respectively. Patients were examined during their visit to a dermatologist (first week) and after applied therapy (sixth week). The severity of the clinical picture was presented in three categories: moderate symptoms, occasional outbreak of changes in rosacea and worsening of rosacea. Following an examination by a dermatologist, the subjects were referred to the Central Laboratory of the University Clinical Centre of Republic of Srpska, where a single blood test was performed for presence of HP infection, and tests for qualitative in vitro detection of IgG antibodies to HP (enzyme-linked immunosorbent assay, ELISA test) were used for this purpose. Laboratory blood analyses were performed by one laboratory technician, and the interpretation, examination and monitoring of the patient were performed by one dermatologist. Based on the positivity of the results concerning the presence of antibodies to HP, the subjects were divided into two groups. Group I consisted of subjects 
in whom, in addition to rosacea, as a primary disease, the presence of HP was registered, while group II consisted of subjects in whom, in addition to rosacea, as a primary disease, no presence of HP was registered, which was also the control group. Topical therapy (metronidazole cream 2 times a day for six weeks, with regular skin hydration using neutral creams) as well as triple therapy for eradication of HP (azithromycin tablets $500 \mathrm{mg} 1 \times 2$, for three days, metronidazole tablets $400 \mathrm{mg} 3 \times 1$, for seven days, pantoprazole tablets $20 \mathrm{mg} 2 \times 1$, for seven days, followed by $1 \mathrm{x}$ $20 \mathrm{mg}$, for 15 days) were administered for group I patients. Only the topical therapy (metronidazole cream $2 \mathrm{x}$ daily for six weeks, with regular hydration using neutral creams) was administered for group II patients.

Statistical processing was performed in the IBM SPSS Statistics 21 software package. The results were presented numerically and tabularly. The $\chi^{2}$ test and the two-sided two-sample test were used for statistical analysis, and the reliability value of 0.05 was taken for the margin of statistical significance.

\section{Results}

The sample of this study consisted of 60 patients diagnosed with rosacea. All subjects were familiar with the study concerned and signed an informed consent. With regard to gender distribution, 52 subjects (86.7\%) were female and 8 subjects $(13.3 \%)$ were male. The age of the subjects ranged from 18 to 79 , with the average age of 45.88. The majority of subjects had skin type according to Fitzpatrick III (91.7 \%) and $8.3 \%$ of patients had type IV. The duration of rosacea was measured in months and ranged from one month to 360 months (30 years), with the average duration of the disease being about 30 months. The general symptoms in the region of the affected skin, included, most frequently, burning (35 subjects or $58.3 \%$ ), itching in 20 subjects (33.3\%) and pain was present in 12 subjects (20\%). Erythema was present in 59 subjects (98.3\%). Papules were present in 47 subjects (78.3\%). Erythema and erythematous papules were observed in 55 subjects ( $91.7 \%$ ), while the pustular stage was registered in 23 subjects (38.3\%). Stage 4 or phymatous rosacea was observed in 6 subjects (10\%).
A qualitative in vitro analysis of IgG antibodies to HP from the blood of patients, registered the presence of HP in 27 patients (45\%), in addition to rosacea as the primary disease (group I), while in 33 patients $(55 \%)$ no presence of these antibodies was registered (group II). As regards the gender distribution, in both examined groups there were more female subjects $-77.7 \%$ in group I and $93.9 \%$ in group II. When it comes to age distribution, a difference was observed, because the majority of patients from group I (55\%) were 18 to 30 years old, while in group II the largest percentage were persons of older age (51-80 years of age). Use of the $\chi^{2}$ test revealed no statistically significant relationship between the frequency of HP infection and the age of the subjects (Table 1).

Table 1: Frequency of Helicobacter pylori infection in relation to the age of the subjects ( $\chi^{2}$ test)

\begin{tabular}{|c|c|c|c|c|c|c|}
\hline \multirow{3}{*}{ Age (years) } & \multirow{3}{*}{$\mathbf{N}$} & \multicolumn{4}{|c|}{ Helicobacter pylori } & \multirow{3}{*}{$p$} \\
\hline & & \multicolumn{2}{|c|}{ No } & \multicolumn{2}{|c|}{ Yes } & \\
\hline & & $N$ & $\%$ & $N$ & $\%$ & \\
\hline $18-30$ & 20 & 9 & 45 & 11 & 55 & \multirow{3}{*}{0.405} \\
\hline $31-50$ & 17 & 9 & 52.9 & 8 & 47 & \\
\hline $51-80$ & 23 & 15 & 65.2 & & 34.8 & \\
\hline
\end{tabular}

N: number of patients;

It was noticed, based on a comparison of the presence of skin changes in the examined groups, that erythema was present in all patients of group I (100\%), and in 32 subjects in group II $(96.9 \%$ of cases). Papules were present in 26 subjects (96.3\%) of group I, and in group II papules were observed in 27 (87.9 \%) subjects. Pustules were present in 15 subjects of group I (55.6\%), and in 8 subjects ( $24.2 \%)$ in group II. No statistical significance was observed between the presence of erythema and papules in relation to the presence of HP infection in the present subjects. However, the application of the $\chi^{2}$ test revealed a statistically significant relationship between the presence of HP infection and the presence of pustules in patients with rosacea $(p=0.027)$, with the incidence of pustules being significantly higher in group I patients than in group II patients. Phyma was present in 1 subject of group I (3.7 \%) and in 5 subjects of group II (15.2\%).

The three registered stages of severity of rosacea symptoms were analysed. It can be noticed that moderate symptoms were more common in group II patients. Worsening of rosacea symptoms was much more common in subjects in the group with HP infection presence (40.7\%). There was no statistically significant difference in the clinical picture severity in relation to the presence of HP infection (Table 2). 
Table 2: Severity of the clinical picture of rosacea in relation to HP infection ( $\chi^{2}$ test)

\begin{tabular}{|c|c|c|c|c|c|c|c|c|}
\hline \multirow{3}{*}{$\begin{array}{l}\text { Helicobater } \\
\text { pylori }\end{array}$} & \multirow{3}{*}{$\mathbf{N}$} & \multicolumn{6}{|c|}{ Rosacea severity } & \multirow{3}{*}{$p$} \\
\hline & & \multicolumn{2}{|c|}{$\begin{array}{l}\text { Moderate } \\
\text { symptoms }\end{array}$} & \multicolumn{2}{|c|}{$\begin{array}{c}\text { Occasional } \\
\text { outbreak }\end{array}$} & \multicolumn{2}{|c|}{$\begin{array}{l}\text { Worsening } \\
\text { of rosacea }\end{array}$} & \\
\hline & & $N$ & $\%$ & $N$ & $\%$ & $N$ & $\%$ & \\
\hline Negative & 33 & 21 & 63.6 & 7 & 21.2 & 5 & 15.2 & \multirow{2}{*}{0.083} \\
\hline Positive & 27 & 12 & 44.4 & 4 & 14.8 & 11 & 40.7 & \\
\hline
\end{tabular}

Of the total number of subjects, erythema regression occurred in 59 subjects ( $98.3 \%$ ), and regression of papules occurred in 53 subjects (88.3\%). Regression of pustules occurred in 23 subjects (38.3\%), and a decrease in phyma was observed in 3 subjects (5\%).

The applied triple therapy for HP infection eradication and the topical therapy for rosacea led to a more pronounced regression of skin changes in group I subjects. Regression of erythema occurred in all 27 subjects (100\%). Papules that were present in 26 group I subjects also receded in all 26 subjects (100\%). In group I, pustules were observed in 15 subjects in the first week, and regression of pustules occurred in 13 subjects (86.7 \%). Regression of skin changes in subjects with phyma was reported.

In group II subjects, erythema regression occurred in all 32 subjects (100\%) in whom erythema was observed in the first week. Also, all subjects with the presence of papules reported regression of changes. During the first week, pustules were observed in 8 subjects, while regression occurred in 5 subjects (62.5\%). Regression of skin changes was observed in 2 subjects ( $40 \%$ ) with phyma.

Table 3: Effectiveness of the applied therapy on pustule and phyma regression (two-sided two-sample test)

\begin{tabular}{|c|c|c|c|}
\hline Skin changes & $\begin{array}{l}\text { Group I } \\
\text { (27 subjects) }\end{array}$ & $\begin{array}{l}\text { Group II } \\
\text { (33 subjects) }\end{array}$ & $p$ value \\
\hline Pustules $1^{\text {st }}$ week & 15 & 8 & \\
\hline $\begin{array}{l}\text { Regression of } \\
\text { pustules } 6^{\text {th }} \text { week }\end{array}$ & $13(86.7 \%)$ & $5(62.5 \%)$ & $p=0.209$ \\
\hline Phyma $1^{\text {st }}$ week & 1 & 5 & \\
\hline $\begin{array}{l}\text { Regression of } \\
\text { phyma } 6^{\text {th }} \text { week }\end{array}$ & $1(100 \%)$ & $2(40 \%)$ & $p=0.006$ \\
\hline
\end{tabular}

Regression of erythema and papules occurred in all subjects of both groups. Table 3 shows the effectiveness of the applied therapy solely with regard to the regression of pustules and phyma.
A comparison of the therapy effectiveness between group I and group II subjects, using a two-sided two-sample test, showed no statistically significant difference in the therapeutic response in pustule regression $(p=0.209)$. As regards the regression of the phyma, there is statistical significance $(p=0.006)$ in favour of group I. However, due to the small number of subjects with the presence of the phyma, these data cannot consider as valid.

\section{Discussion}

In this study, of the total number of patients, 86.7 $\%$ were female patients and $13.3 \%$ were male patients, while their age ranged from 18 to 79 years, with the average age of 45.88. Of the total 27 number of subjects, who were positive for HP infection most of which were women $(77.7 \%)$, while only 6 subjects (22.3\%) were men. HP infection was somewhat more frequent in younger subjects, ie, the presence of HP infection was observed in $55 \%$ of subjects aged 18-30.

Szlachcic established a close link between HP infection and the appearance of rosacea. He states that HP can cause skin inflammation in two ways: by increasing the concentration of nitric oxide (consequently vasodilation) and by inducing a specific cytotoxic reaction, ie, the expression of cytotoxic genes and initiation of a series of inflammatory reactions. ${ }^{3}$ On the other hand, based on a quantitative analysis of fourteen studies involving 928 patients and 1527 controls, Jørgensen et $\mathrm{al}^{21}$ concluded that there was a weak link between HP infection and rosacea, just like that the application of the triple therapy to eradicate HP infection does not result in a significant regression of rosacea symptoms, which is contrary to the results obtained in this studied sample. Yang recommends that all patients with rosacea should be tested for the presence of HP infection in order to achieve the desired therapeutic effects ${ }^{22}$ by eradicating the infection, which would be the recommendation of the present study's result, too.

There are theories suggesting that HP infection is a predisposing factor for the appearance of rosacea. Hong observed a more frequent rate of HP infection (Urase-IgG antibodies and HP CagA-IgG antibodies) in the peripheral blood of 39 patients 
with rosacea. The incidence rate in patients with rosacea and digestive tract symptoms was $86.7 \%$, and in patients with rosacea without digestive tract symptoms the incidence rate was $16.7 \%{ }^{23}$

The dominant symptom in these patients include burning (58.3\%), itching in $33.3 \%$ and pain as a general symptom of rosacea in $20 \%$. Given the present skin changes, erythema was most common (98.3\% of subjects), followed by erythema papules in $91.7 \%$, while the third stage or pustular stage was registered in $38.3 \%$ of subjects, and the fourth stage or phymatous rosacea in $10 \%$.

Skin changes in these subjects show that there is a statistically significant relationship between the presence of HP infection and the presence of pustules in patients with rosacea, with the incidence of pustules being significantly higher in patients with HP infection $(55.6 \%)$ than in patients without infection $(24.2 \%)$. The severity of the clinical picture of rosacea with regards to HP infection shows that moderate symptoms are more common in patients without HP infection (63.6 \%) compared to positive subjects (44.4\%), while worsening of rosacea symptoms is much more common in subjects with present HP infection $(40.7 \%)$ than in subjects with rosacea without HP infection (15.2\%).

The applied triple therapy for eradicating HP infection and topical therapy for rosacea in the group I patients led to a more pronounced regression of skin changes, compared to the application of topical therapy in group II patients, but without confirmed statistical significance. The results are in accordance with the data from the literature. ${ }^{21-23}$ Piri showed the success of standard triple therapy for eradicating the bacterium but emphasises that due to the high resistance of the bacterium to antibiotics, a better and more effective antibiotic therapy is needed to fight HP. ${ }^{24}$

Authors Saleh et al attempted, in a clinical study, to evaluate the effect of a standard HP eradication protocol on the clinical course of rosacea. In this clinical trial, patients diagnosed with HP infection based on serological studies were evaluated as patients that should be tested for rosacea presence, underwent standard HP eradication therapy. Rosacea was evaluated using the Duluth rosacea grading score at the beginning, 2 months later and at the end of the trial (day 180). Of 872 patients positive for HP, 167 patients (19.15\%) manifested the clinical features of rosacea. The patients with concomitant rosacea and the presence of HP were younger and predominantly female, as was the case in the presented sample. Of 167 patients, 150 received HP eradication therapy, demonstrating a $92 \%$ cure rate. The rosacea Duluth score grading on day 0,60 , and 180 among 138 patients significantly decreased in most criteria, except for telangiectasias $(p=0.712)$ and phymatous changes $(\mathrm{p}=0.535)$. This study concluded that eradication of HP leads to improvement in rosacea. ${ }^{25}$ The results of the present study are in line with the data of this study.

Contrary to the majority of studies, Herr states that he did not observe a significant improvement in skin changes in rosacea applying triple therapy. Namely, he examined 100 patients with rosacea, but 50 of them received triple therapy, while the other 50 patients received placebo. It was observed that after 2 months the intensity of regression of changes in both groups was approximately the same. ${ }^{26}$ The results of this study are consistent with the conclusions of Jorgensen et al. ${ }^{21}$

However, it is necessary to emphasise that there are limitations to the test for HP presence using blood analysis (tests for qualitative in vitro detection of IgG antibodies to HP). Namely, this test does not detect the difference between the current and a previous infection. For purposes of accuracy of testing, the new recommendations refer to the method of detection of HP from a stool sample, rather than a blood sample, as a more reliable one.

\section{Conclusion}

In this study sample, the presence of HP infection was registered in $45 \%$ of patients, more frequently in younger subjects and females. The pustular form of rosacea was more common in patients who tested positive for HP. Worsening of rosacea symptoms was much more common in subjects with HP infection. Therapy for eradicating HP infection improves the clinical course of rosacea, especially in the stages of papules and pustules. 


\section{Acknowledgements}

None.

\section{Conflict of interest}

None.

\section{References}

1. Culp B, Scheinfeld N. Rosacea: A review. P T 2009 Jan;34(1):38-45.

2. Goldberg DJ, Berlin AL. Rosacea - epidemiology and pathophysiology. In: Goldberg DJ, Berlin AL. Acne and rosacea. Epidemiology, diagnosis and treatment. London: Manson Publishing, 2013; p. 51-58.

3. Szlachcic A. The link between Helicobacter pylori infection and rosacea. J Eur Acad Dermatol Venereol 2002 Jul;16(4):328-33.

4. Ertl GA, Levine N, Kligman AM. A comparison of the efficacy of topical tretinoin and low-dose oral isotretinoin in rosacea. Arch Dermatol 1994 Mar;130(3):319-24.

5. Agnoletti AF, DE Col E, Parodi A, Schiavetti I, Savarino V, Rebora A, et al. Etiopathogenesis of rosacea: a prospective study with a three-year follow-up. G Ital Dermatol Venereol 2017 Oct;152(5):418-23.

6. Bhattarai S, Agrawal A, Rijal A, Majhi S, Pradhan B, Dhakal SS. The study of prevalence of Helicobacter pylori in patients with acne rosacea. Kathmandu Univ Med J (KUMJ) 2012 Oct-Dec;10(40):49-52.

7. Abadi ATB. Strategies used by Helicobacter pylori to establish persistent infection. World J Gastroenterol 2017 Apr 28;23(16):2870-82.

8. Saleh P, Naghavi-Behzad M, Babapour S, Piri R. The association between Helicobacter pylori infection and rosacea. Arch Clin Infect Dis 2018;13(1):e57740. DOI : 10.5812/ archcid.57740.

9. Lazaridou E, Korfitis C, Kemanetzi C, Sotiriou E, Apalla Z, Vakirlis E, et al. Rosacea and Helicobacter pylori: links and risks. Clin Cosmet Investig Dermatol 2017;10:305-10.

10. Kubanov A, Gallyamova Y, Kravchenko A. Clinical picture, diagnosis and treatment of rosacea, complicated by Demodex mites. Dermatol Reports 2019 Mar 28;11(1):7675. doi: 10.4081/dr.2019.7675.

11. Gonser LI, Gonser CE, Schaller M. [Erratum to: Pathogenesis, clinical picture, and current therapy of rosacea]. Hautarzt 2016;67(5):384. German. doi: 10.1007/s00105016-3786-2.

12. Logger JGM, de Vries FMC, van Erp PEJ, de Jong EMGJ, Peppelman M, Driessen RJB. Non-invasive objective skin measurement methods for rosacea assessment: a systematic review. Br J Dermatol 2020 Jan;182(1):55-66.
13. Egeberg A, Weinstock LB, Thyssen EP, Gislason GH, Thyssen JP. Rosacea and gastrointestinal disorders: a population-based cohort study. Br J Dermatol 2017 Jan;176(1):100-6.

14. Olazagasti J, Lynch P, Fazel N. The great mimickers of rosacea. Cutis 2014 Jul;94(1):39-45.

15. Tüzün Y, Wolf R. Red face revisited: II. Clin Dermatol 2014 Nov-Dec;32(6):709-10.

16. Rivero AL, Whitfeld M. An update on the treatment of rosacea. Aust Prescr 2018 Feb;41(1):20-4.

17. Baldwin HE. Systemic therapy for rosacea. Skin Therapy Lett 2007 Mar;12(2):1-5, 9.

18. Kubanov A, Gallyamova Y, Kravchenko A. Clinical picture, diagnosis and treatment of rosacea, complicated by Demodex mites. Dermatol Reports 2019 Mar 28;11(1):7675. doi: 10.4081/dr.2019.7675.

19. Griffiths C, Barker J, Bleiker T, Chalmers R, Creamer D. Rook's Textbook of Dermatology 9th edition. London: John Wiley \& Sons Ltd, 2016; p.1851-1912.

20. Kong S, Amangai M, Bruckner A, Enk A, Margolis D, McMichael A, et al. Fitzpatrck's dermatology in general medicine, 9th edition. New York: Mc Graw Hill Education Europe, 2019.

21. Jørgensen AR, Egeberg A, Gideonsson R, Weinstock LB, Thyssen EP, Thyssen JP. Rosacea is associated with Helicobacter pylori: a systematic review and meta-analysis. J Eur Acad Dermatol Venereol 2017 Dec;31(12):2010-5.

22. Yang X. Relationship between Helicobacter pylori and rosacea: review and discussion. BMC Infect Dis $2018 \mathrm{Jul}$ 11;18(1):318. doi: 10.1186/s12879-018-3232-4.

23. Hong J. Incidence and clinical significance of Helicobacter pylori associated protein antibody in peripheral blood of 39 patients with rosacea. Gui Zhou Med 2014;38(6):541-2.

24. Piri R. Comment on "Effects of Helicobacter pylori treatment on rosacea: A single-arm clinical trial study". J Dermatol 2017 Dec;44(12):e370. doi: 10.1111/13468138.13999.

25. Saleh P, Naghavi-Behzad M, Herizchi H, Mokhtari F, Mirza-Aghazadeh-Attari M, Piri R. Effects of Helicobacter pylori treatment on rosacea: A single-arm clinical trial study. J Dermatol 2017 Sep;44(9):1033-7.

26. Herr H, You CH. Relationship between Helicobacter pylori and rosacea: it may be a myth. J Korean Med Sci Oct 2000;15(5):551-4. 